\title{
DSMC simulations of polarized atomic beam sources including magnetic fields
}

\section{Gaisser}

Institut für Kernphysik, Forschungszentrum Jülich, 52425 Jülich, Germany

E-mail: m.gaisserefz-juelich.de

\section{A. Nass*}

Institut für Kernphysik, Forschungszentrum Jülich, 52425 Jülich, Germany

E-mail: a.nass@fz-juelich.de

\section{H. Ströher}

Institut für Kernphysik, Forschungszentrum Jülich, 52425 Jülich, Germany

E-mail: h.stroeherefz-juelich.de

In recent decades a lot of work has been done to understand and optimize the output of polarized atomic beam sources mainly with tracking calculations of atoms moving through the field of the sextupole magnet system with the measured parameters of the supersonic atomic beam as starting conditions. Besides of these simple calculations, attempts were made to achieve an analytical description of the processes in an atomic beam source. However, there are many effects which prevent a complete analytic description of the system. A new DSMC (Direct Simulation Monte Carlo) simulation based on OpenFOAM 1.7.1 using Birds algorithm has been developed which calculates the output intensity including all major processes occuring in the atomic beam source, e.g., influence of magnetic fields, high frequency transitions, rest gas scattering, recombination, intra-beam scattering, and spin exchange collisions.

So far, the simulated particles have been given spin and a generic utility to include arbitrary magnetic fields has been created. The equation of motion in every timestep is solved by a fourth order Runge-Kutta scheme. High frequency transition units are included as well as recombination on the walls and spin exchange collisions. Additionally, a generic interface for optimization algorithms was built and Adaptive Simulated Annealing was put into this framework to optimize the output of DSMC simulations. Last but not least, a tool to measure the collision age of particles in a storage cell has been created and first tests of the program are promising.

This development is in particular important for the PAX project (Polarized Antiproton eXperiments) since a high-density highly polarized target is necessary in order to effectively polarize a stored antiproton beam.

XVth International Workshop on Polarized Sources, Targets, and Polarimetry,

September 9-13, 2013

Charlottesville, Virginia, USA

\footnotetext{
* Speaker.
} 


\section{Introduction}

The high physics potential of experiments with stored polarized antiprotons led to the proposal of the PAX [1] experiment for an upgrade of the High Energy Storage Ring (HESR) of the new FAIR facility under construction at GSI (Darmstadt/Germany). It is proposed to polarize a stored antiproton beam by means of spin filtering by a polarized hydrogen gas target.

The PAX polarized target consists of a polarized Atomic Beam Source (ABS) [2], a storage cell and a diagnostic system. The polarized atomic hydrogen beam from the ABS is injected into the storage cell where hundreds of wall bounces lead to an increase of the target thickness. The polarization of a gas sample from the storage cell is analyzed in the diagnostic system which consists of a Target Gas Analyzer (TGA) and the so-called Breit-Rabi Polarimeter (BRP). A set of coils mounted on the scattering chamber provides the weak holding field $(\approx 10 \mathrm{G})$ to orient the target polarization. The intensity of the atomic beam source was $I=6.6 \cdot 10^{16}$ atoms/s leading to a target thickness of $d_{t}=(5.5 \pm 0.2) \cdot 10^{13}$ atoms $/ \mathrm{cm}^{2}$, measured using the beam energy loss method. The target gas polarization was deduced by the TGA and BRP to be $Q=0.73 \pm 0.05$.

A high target thickness and polarization are crucial in order to increase the polarization of the antiproton beam and to reduce the filtering time. To achieve higher intensities of the ABS a lot of effort was put into the understanding and optimization of the output of polarized atomic beam sources (e.g. [3]). But there are many effects which prevent a complete analytic description of the system. Recently, a DSMC simulation [4] based on OpenFOAM 1.7.1 using Birds [5] algorithm was created.

\section{DSMC}

In order to calculate or simulate a gas stream the local density is an important quantity which defines the type of flow occurring. The Knudsen number $K n=\frac{\lambda}{L}$ can be defined for all gases, where $L=\frac{\rho}{|\nabla \rho|}$ is a locally defined length scale. For so-called rarefied gases with $K n>0.1$ the NavierStokes equation doesn't hold anymore since the coupling between neighboring volume elements is not strong enough. Molecular flows are described by the Boltzmann equation where the flux of particles in and out of volume elements is considered. This integro-differential equation is not solvable analytically for practical cases and even the numerical handling is extremely difficult. Birds DSMC procedure [5] effectively circumvents this equation by directly simulating some of the gas atoms or molecules. It was shown that the Boltzmann equation can be derived from the DSMC procedure and that the method converges in some limits towards a solution of the Boltzmann equation. The basic assumption of the DSMC method is that the motion of the particles and the collisions are decoupled on short time scales, i.e. on time scales much less than the mean collision time the gas particles can be moved along straight lines without collisions. After all particles are moved for a certain time step collision partners will be chosen randomly from within a certain volume element. This requires a partitioning of the computational volume into small cells which is generally called a mesh. The size of the mesh cells should be smaller than the mean free path length of the gas particles and ideally about 20 simulated particles should be within such a cell. The sequence of tasks during a simulation is then as follows: Read or create a mesh of the computational volume and fill the mesh cells with particles according to the initial and boundary conditions. In the 
main loop all particles are moved for a fixed time step and certain boundary conditions are applied, e.g. if the particles hit a wall during the time step. After all particles are moved, collision partners are chosen from within a cell or subcell and the post-collision velocities are calculated according to some collision model. Now the particles are moved again with the new velocities or the fields (e.g. temperature, pressure etc.) can be calculated and written to file. The program finally ends if a user specified simulated time is reached.

As starting point for all the work OpenFOAM version 1.7.1 available from www.openfoam.org was chosen together with the extension dsmcModFoam available from www.physimmasters.com. This program is written in $\mathrm{C}++$, has lots of pre- and post-processing capabilities and is intended for user written extensions. It can be run in parallel, either on a local workstation or distributed over several computers. The parallelization paradigm is domain decomposition, meaning that certain subsets of mesh cells are assigned to the various processors. An example of a simulation of an ABS is shown in figure 1. A comparison of simulated and measured $[3,6]$ values is shown in table 1. The simulated beam parameters are in good agreement with the measured ones. Considering the position difference of the pressure gauges, especially in the vacuum chambers 1 and 2, compared with the location in the simulated space, also the pressures are in good agreement with the measurements.
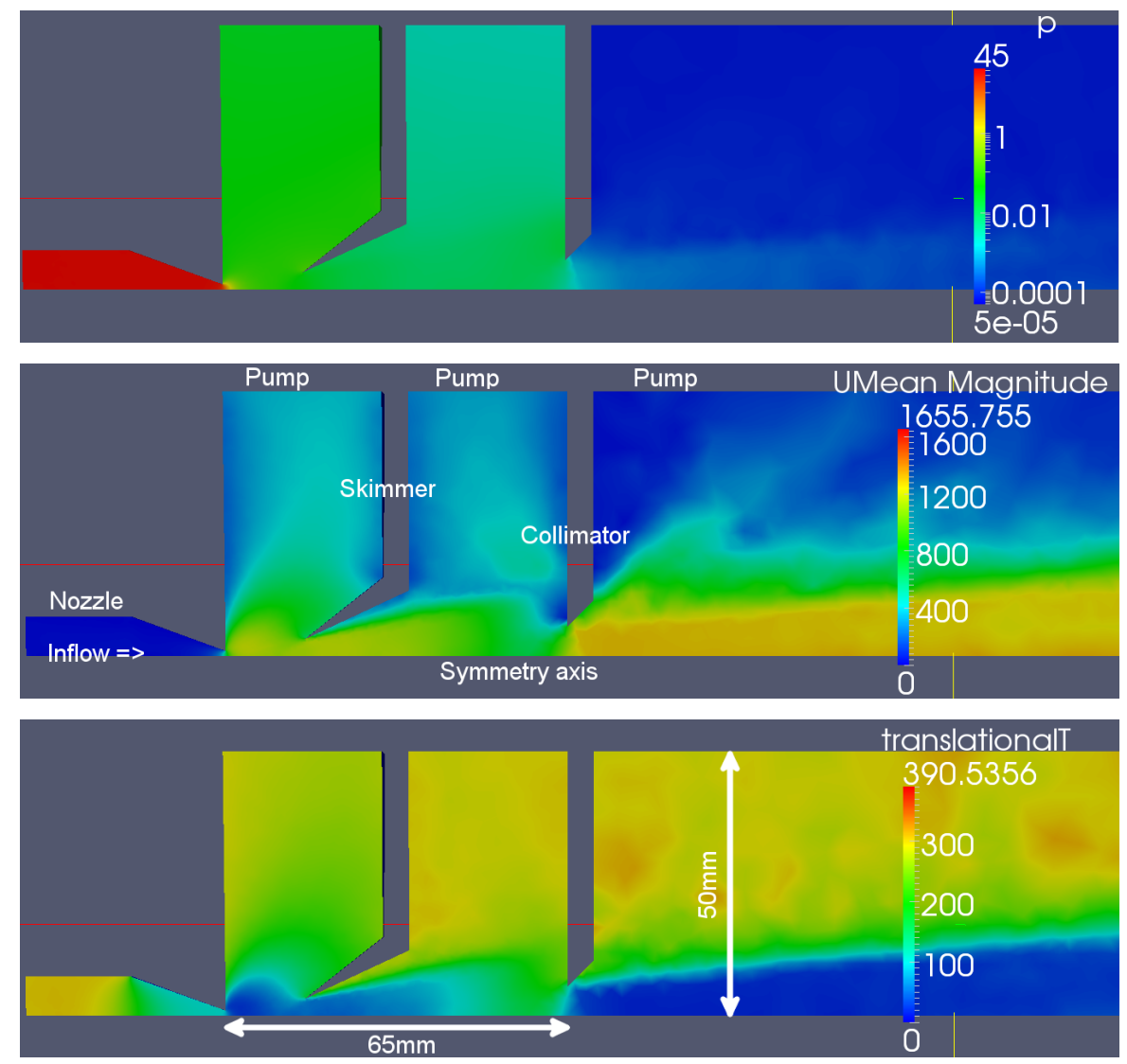

Figure 1: Pressure (top), mean velocity (middle) and translational beam temperature (bottom) distribution, in the first part of an ABS. Simulated is a flux of $1 \mathrm{mbarl} / \mathrm{s} \mathrm{H}_{2}$ with $300 \mathrm{~K}$ and a nozzle temperature of $100 \mathrm{~K}$. 


\begin{tabular}{|l|l|l|}
\hline & measured & simulated \\
\hline$U_{\text {mean }}[\mathrm{m} / \mathrm{s}]$ & $1274 \pm 8.4$ & 1290 \\
$T_{\text {trans }}[\mathrm{K}]$ & $19.03 \pm 1.11$ & 16.5 \\
$p_{1}$ & $2.1 \pm 0.2 \cdot 10^{-4}$ & $3.4 \cdot 10^{-4}$ \\
$p_{2}$ & $1.8 \pm 0.2 \cdot 10^{-5}$ & $2.7 \cdot 10^{-5}$ \\
$p_{3}$ & $7.0 \pm 0.7 \cdot 10^{-7}$ & $8.0 \cdot 10^{-7}$ \\
$p_{4}$ & $1.7 \pm 0.2 \cdot 10^{-7}$ & $1.7 \cdot 10^{-7}$ \\
\hline
\end{tabular}

Table 1: Measured and simulated beam parameters and chamber pressures for a flux of $1 \mathrm{mbarl} / \mathrm{s} \mathrm{H}_{2}$ with $300 \mathrm{~K}$ and a nozzle temperature of $100 \mathrm{~K}$.

Collision Models In ref. [6] it was shown with Birds DSMC code that simulated results are in good agreement with measurements at a test bench, except for the simulated temperatures. This can be attributed to the usage of the inverse power law potential for inter-particle forces. Real molecules have a long range attractive part of the potential which is especially effective at low collision energies, i.e. low gas temperatures. Therefore a Variable Soft Sphere (VSS) collision model based on the Lennard-Jones potential was implemented as proposed in ref. [7]. Since the necessary cross sections cannot be given explicitely, fit functions were used. For inelastic collisions with energy exchange between translational and internal (rotational, vibrational) degrees of freedom the phenomenological Larsen-Borgnakke model is used where only a fraction of the collisions is considered as inelastic to match the calculated and observed relaxation rates. For the Lennard-Jones potential the necessary probability distributions cannot be reasonably applied, and for the moment the ones from the inverse power law model are used. The effect from this for simulations of an ABS is supposed to be small. The data in table 1 was generated with a collision model using a Lennard-Jones potential.

Spin is included in the simulated atoms in their ground state with electron spin $1 / 2$ and nuclear spin $\leq 3 / 2$ which is sufficient for $\mathrm{H}$ and $\mathrm{D}$. For visualization and calculation of the polarization in homogenous magnetic fields arrays have been created which show the relative population number for each hyperfine state.

Magnetic Fields are established in the DSMC code as a generic interface. For every magnet type there has to be a $\mathrm{C}++$ class that specifies the magnetic field and its gradient at a given position and a function that shows where the field is active. Then, the user has the possibility to create many magnets of this type with different parameters (size, field strength and orientation) via a configuration file. The program allows for arbitrary superpositions of magnetic fields and the equation of motion for the particles is solved with a fourth order Runge-Kutta scheme. In magnetic fields, curved tracks in combination with the standard tracking of particles along straight lines lead to errors such as false wall hits and systematic shifts in energy and position. This made it necessary to depart from the standard algorithm using straight lines for the trajectory. Now the trajectory is approximated by a parabola fitted through the points $\mathbf{x}(t=0), \mathbf{x}(t=\Delta t / 2)$ and $\mathbf{x}(t=\Delta t)$ and the intersection of this parabola with the cell faces is calculated. The error from this method is of order $O\left(\Delta t^{4}\right)$ and is smaller than $10^{-10} \mathrm{~m}$ even for big time steps of $\Delta t=10^{-6} \mathrm{~s}$. Another issue arises 
from idealizing the magnetic field to be active only inside of magnet bores. Thus there is a surface at the magnet entrance where the field drops from a certain value to zero and the gradient is infinite. Considering the difference of potential energy on both sides of this surface shows, that this is in general not negligible and hence a correction to the velocity normal to this surface is applied.

Recombination occurs with some statistical probability mainly on the walls of the containment vessel since volume recombination is suppressed due to the low gas densities. Atoms from the gas phase can recombine with atoms adsorbed on the walls. The description of the underlying processes is very complicated. The recombination probability depends strongly on the surface coverage which in turn depends on the gas and wall temperatures, the gas density and the surface properties. The implementation in the DSMC program was done using a simplified model with a fixed recombination probability for every wall patch, with respect to different material and/or temperature of the walls. An example of a simple DSMC calculation with particles in a box can be seen in Fig. 2. As expected the atoms recombine into molecules with conservation of the number of hydrogen nuclei.

Spin exchange collisions are possible if two hydrogen (deuterium) atoms collide. A short-living bound state might be formed and the interaction can cause an exchange of electron and nuclear spin. Due to angular momentum conservation, the population of hyperfine states cannot change arbitrarily. In the DSMC calculations the cross section for spin exchange collisions is taken to be the same as for momentum collisions. The probability of a spin exchange in such a collision which depends on the magnetic field can be found in ref. [8]. An example of a simple DSMC calculation with particles in a box can be seen in Fig. 3 .

High Frequency Transitions (HFT) deliberately exchange occupation numbers of hyperfine levels. A HFT composes of a static magnetic field (with a spatial gradient) with an RF-field whose

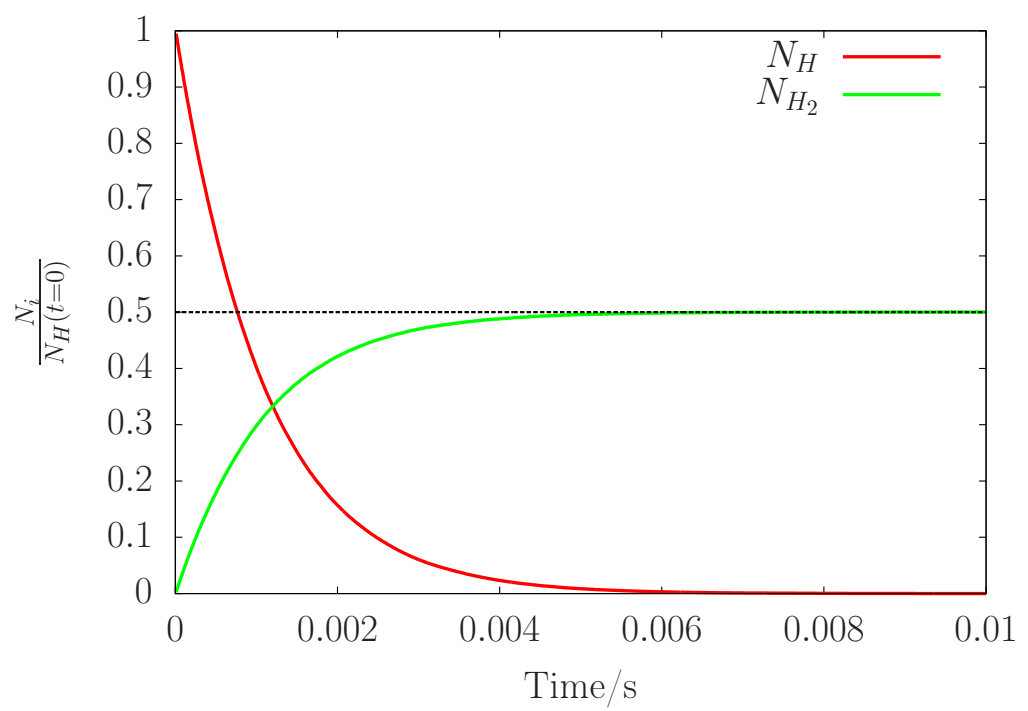

Figure 2: Relative numbers of hydrogen atoms and molecules in a closed box with recombination on the box walls only. 

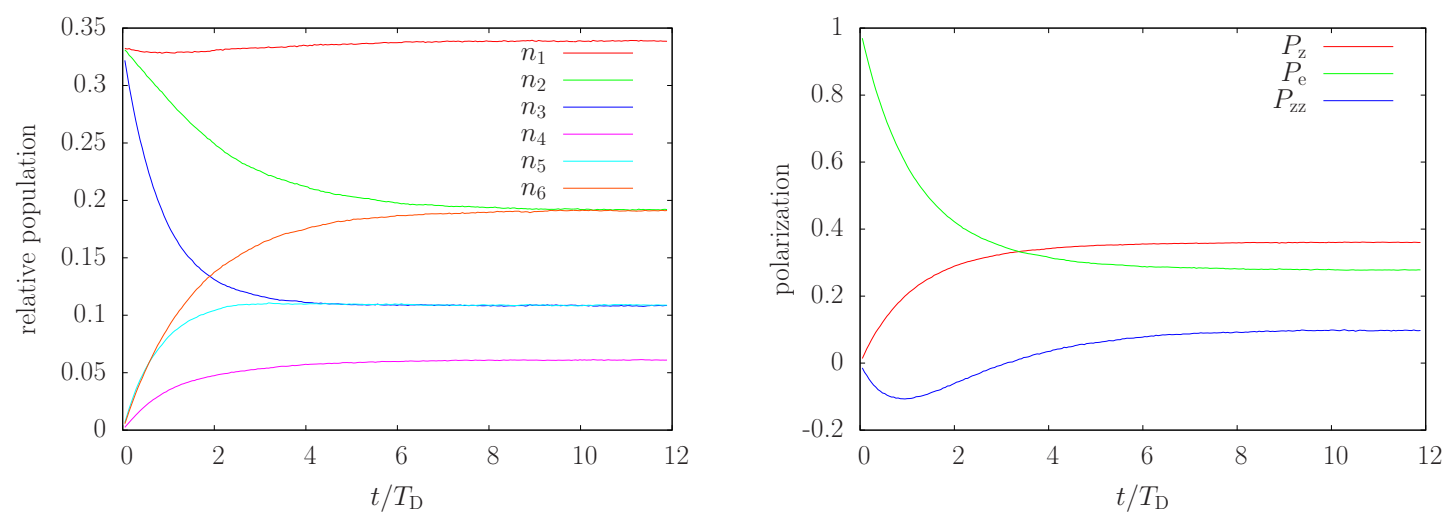

Figure 3: Occupation numbers of the respective hyperfine states and polarization of an ensemble of deuterium atoms in a closed box regarding spin exchange collisions only.

frequency matches the energy difference between two hyperfine levels to transfer an atom from state $|a\rangle$ to $|b\rangle$. That way the output polarization of an ABS can be changed. Efficiencies of HFT are usually high [2]. In the DSMC code HFT units are idealized such that all transitions take place in one plane. If a particle crosses that plane, its hyperfine state will be changed with a certain probability into another hyperfine state. The plane can be chosen arbitrarily independent of the mesh.

Collision age calculations are necessary to investigate the influence of wall collisions in a storage cell. To measure the polarization, parts of the gas are extracted from the storage cell through a long narrow tube to the polarimeter. Along this tube the particles collide with the wall and can recombine and depolarize. In order to relate the measured polarization with the one occurring in the storage cell, it is important to know the depolarization rate which scales with the number of wall collisions. A first simulation of a storage cell (Fig. 4) reproduces the shape of a theoretically expected curve for the collision age. It should be noted, that this can also be achieved with simpler and faster ray tracing codes but the current program can also be used for denser gases where the number of particle-particle collisions is not negligible. This tool is also supposed to help fitting parameters that will be used to describe recombination and depolarization on the walls.

Intensity optimization of atomic beam sources The target thickness is crucial for future experiments with polarized internal gas targets. An optimization algorithm was developed in order to maximize the output intensity of an ABS. This algorithm is based on the Adaptive Simulated Annealing mechanism (formerly Very Fast Simulated Re-Annealing [9]). The result of a so-called cost function $f$ (the output intensity in our case) is calculated for a model ABS with $n$ parameters. After that the parameters are modified using a certain distribution with a control parameter $T_{\mathrm{a}}$ around the start value. A new result of $f$ is calculated and accepted with a defined probability which is higher for higher intensities than for lower ones. This process is repeated and $T_{\mathrm{a}}$ is reduced with the number of repetitions. The distribution of new parameters assures that the algorithm does not get stuck in a local maximum, since new values in the complete parameter space are possible although less probable with increasing number of repetitions. The method was shown to converge to the global maximum. 


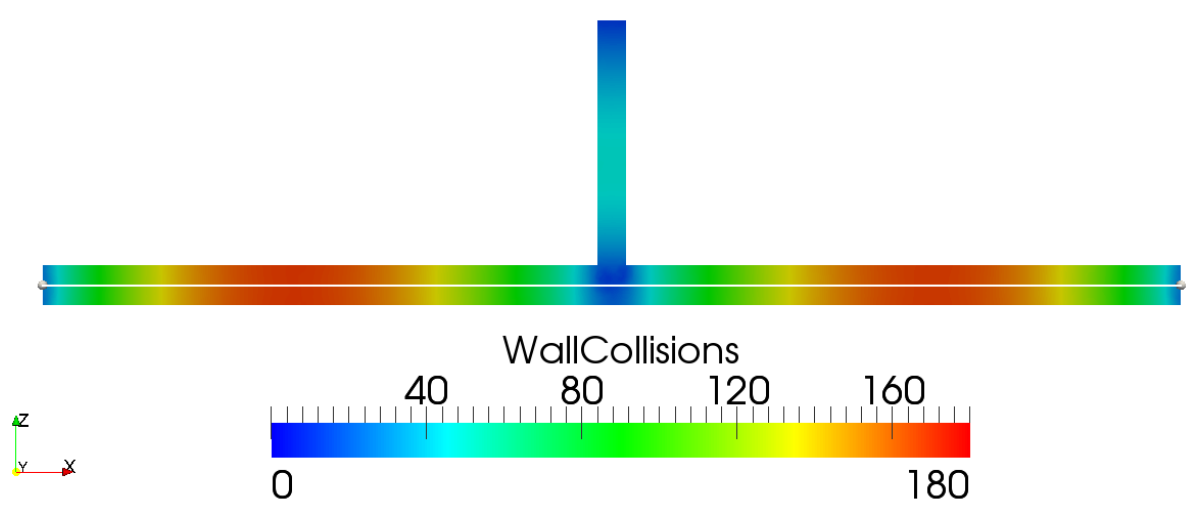

Figure 4: Distribution of the wall collision age inside the PAX storage cell. The average collision age is 116.5 wall bounces.

\section{Summary and Outlook}

It was shown that the DSMC calculations based on OpenFOAM 1.7.1 with the additional features described above individually reproduce the processes occurring in an ABS. The current task is to simulate a complete ABS and compare the results with the measurements. After a successful simulation the optimization algorithm will be used to define the parameters for future high intensity atomic beam sources.

\section{References}

[1] Antiproton-Proton Scattering Experiments with Polarization, Technical Proposal for the HESR at FAIR, Jülich (2005), e-Print Archive: hep-ex/0505054.

[2] A. Nass et al, Nucl. Instr. Meth. A 505 (2003) 633-644.

[3] N. Koch, A Study on the Production of Intense Cold Atomic Beams for Polarized Hydrogen and Deuterium Targets, PhD-thesis (1999) ISSN 1435-8085.

[4] T. J. Scanlon et al, Computers \& Fluids 39 (2010) 2078.

[5] G. A. Bird, Molecular Gas Dynamics and the Direct Simulation of Gas Flows, Oxford Science Publications, 1998.

[6] A. Nass and E. Steffens, Nucl. Instr. Meth. A 598 (2009) 653-666.

[7] K. Koura and H. Matsumoto, Phys. FluidsA 3 (1991) 2459.

[8] T. Walker and L. Anderson, Nucl. Instr. Meth. A 334 (1994) 313.

[9] L. Ingber, Mathl. Comput. Modeling, Vol. 12, No. 8, (1989) 967-973. 